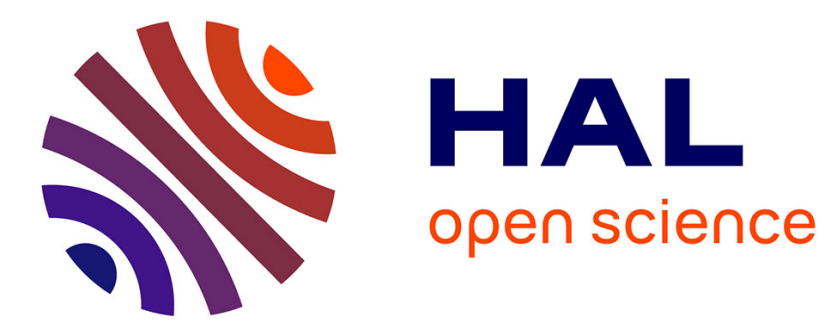

\title{
Interface response theory of composite elastic media
}

\author{
L. Dobrzynski, J. Mendialdua, A. Rodriguez, S. Bolibo, M. More
}

\section{To cite this version:}

L. Dobrzynski, J. Mendialdua, A. Rodriguez, S. Bolibo, M. More. Interface response theory of composite elastic media. Journal de Physique, 1989, 50 (18), pp.2563-2578. 10.1051/jphys:0198900500180256300 . jpa-00211082

\section{HAL Id: jpa-00211082 https://hal.science/jpa-00211082}

Submitted on 1 Jan 1989

HAL is a multi-disciplinary open access archive for the deposit and dissemination of scientific research documents, whether they are published or not. The documents may come from teaching and research institutions in France or abroad, or from public or private research centers.
L'archive ouverte pluridisciplinaire HAL, est destinée au dépôt et à la diffusion de documents scientifiques de niveau recherche, publiés ou non, émanant des établissements d'enseignement et de recherche français ou étrangers, des laboratoires publics ou privés. 
Classification

Physics Abstracts

$68.25-68.65-68.15-68.48-64.45$

\title{
Interface response theory of composite elastic media
}

\author{
L. Dobrzynski, J. Mendialdua (*), A. Rodriguez (*), S. Bolibo and M. More \\ Equipe Internationale de Dynamique des Interfaces, Laboratoire de Dynamique des Cristaux \\ Moléculaires, U.R.A. No. 801 au CNRS, U.F.R. de Physique, Université de Lille I, F-59655 \\ Villeneuve d'Ascq Cedex, France
}

(Reçu le 2 février 1989, accepté sous forme définitive le 21 mars 1989)

\begin{abstract}
Résumé. - Une théorie générale permettant d'étudier n'importe quel matériau élastique et composite est proposée. Son application aux composites lamellaires est ensuite développée. Ces résultats généraux sont illustrés par des exemples de nouveaux modes de vibration localisés dans des couches fluide et solide comprises entre deux autres solides semi-infinis.
\end{abstract}

\begin{abstract}
A general theory for the study of any composite elastic media is proposed. It is then applied to layered composites. These general results are illustrated by examples of new localized vibrations within fluid and solid slabs sandwiched between two other semi-infinite solids.
\end{abstract}

\section{Introduction.}

Following the pioneering works [1] of I. M. Lifshitz for phonons and J. Friedel for electrons on the study of a point defect in a solid, the surface of a solid was studied as a planar defect in an infinite solid [2]. Interfaces between two different media [3] and then superlattices [4] started then to be considered in a similar manner. However this approach to interface problems requires one to know first the properties of the free surface pieces of matter out of which the composite material is built. It was shown recently [5] that the study of any composite material can be undertaken directly from the bulk properties of each of its pieces together with the boundary conditions at the interfaces. This interface response theory [5] deals in a unified manner with problems formulated within discrete and continuous spaces. It was applied to phonons, magnons, and electrons in the tight binding [6] approximation as well as to electromagnetism and free electrons [7]. The present paper shows how this theory [5] enables any composite elastic media to be studied, and gives new results for localized elastic vibrations within a fluid and a solid slab sandwiched between two other semi-infinite solids.

The next section of this paper gives the general equations enabling any composite elastic material to be studied. Section 3 illustrates this general theory by an application to layered

(*) Permanent address : Departamento de Fisica, Facultad de Ciencias, Universidad de Los Andes, Merida, Venezuela. 
composite materials. Finally, we give specific new results for localized elastic vibrations within a fluid and a solid slab sandwiched between two other semi-infinite solids.

\section{General theory for any elastic composite material.}

2.1 BULK AND FREE SURFACE EQUATIONS. - The equation of motion for the displacements $u_{\alpha}, \alpha=1,2,3$ of a point of an infinite homogeneous 3 -dimensional elastic material is

$$
-\rho \omega^{2} u_{\alpha}=\sum_{\beta} \frac{\partial T_{\alpha \beta}}{\partial x_{\beta}}
$$

where $\rho$ is the mass density, $\omega$ the vibrational frequency, $T_{\alpha \beta}$ the stress tensor

$$
T_{\alpha \beta}=\sum_{\mu \nu} C_{\alpha \beta \mu \nu} \eta_{\mu \nu}
$$

$C_{\alpha \beta \mu \nu}$ are the elastic constants and $\eta_{\mu \nu}$ the deformation tensor

$$
\eta_{\mu \nu}=\frac{1}{2}\left(\frac{\partial u_{\mu}}{\partial x_{\nu}}+\frac{\partial u_{\nu}}{\partial x_{\mu}}\right)
$$

Then the bulk equation of motion (2.1) can be rewritten as

$$
-\rho \omega^{2} u_{\alpha}=\sum_{\beta \mu \nu} \frac{\partial C_{\alpha \beta \mu \nu}}{\partial x_{\beta}} \frac{\partial u_{\mu}}{\partial x_{\nu}}+\sum_{\beta \mu \nu} C_{\alpha \beta \mu \nu} \frac{\partial^{2} u_{\mu}}{\partial x_{\beta} \partial x_{\nu}} .
$$

Suppose now that the elastic matter is limited by a free surface whose position in the infinite 3-dimensional space is given by

$$
x_{3}=f\left(x_{1}, x_{2}\right) \text {. }
$$

Then the elastic constants are

$$
C_{\alpha \beta \mu \nu}(\mathbf{x})=\theta\left[x_{3}-f\left(x_{1}, x_{2}\right)\right] C_{\alpha \beta \mu \nu},
$$

where the Heavside step function is such that

$$
\theta\left[x_{3}-f\left(x_{1}, x_{2}\right)\right]=\left\{\begin{array}{ll}
1 & \text { for } x_{3} \geqslant f\left(x_{1} x_{2}\right) \\
0 & \text { for } x_{3}<f\left(x_{1} x_{2}\right)
\end{array} .\right.
$$

The use in equation (2.6) of $\theta\left[x_{3}-f\left(x_{1}, x_{2}\right)\right]$ means that the elastic medium occupies the space $x_{3}>f\left(x_{1}, x_{2}\right)$.

Define with the help of equations (2.4) and (2.6) the bulk operator

$$
H_{\alpha \mu}(\mathbf{x})=\theta\left[x_{3}-f\left(x_{1}, x_{2}\right)\right]\left(\rho \omega^{2} \delta_{\alpha \mu}+\sum_{\beta \nu} C_{\alpha \beta \mu \nu} \frac{\partial^{2}}{\partial x_{\beta} \partial x_{\nu}}\right)
$$

and the cleavage operator

$$
V_{\alpha \mu}(\mathbf{x})=\sum_{\beta \nu} \frac{\partial C_{\alpha \beta \mu \nu}(\mathbf{x})}{\partial x_{\beta}} \frac{\partial}{\partial x_{\nu}}
$$


such that

$$
\overleftrightarrow{h}=\overleftrightarrow{H}+\overleftrightarrow{V}
$$

Note that after differentiation of the right hand side of equation (2.8b), one has

$V_{\alpha \mu}(\mathbf{x})=+\delta\left[x_{3}-f\left(x_{1}, x_{2}\right)\right] \sum_{\nu}\left[-C_{\alpha 1 \mu \nu} \frac{\partial f\left(x_{1}, x_{2}\right)}{\partial x_{1}}-C_{\alpha 2 \mu \nu} \frac{\partial f\left(x_{1}, x_{2}\right)}{\partial x_{2}}+C_{\alpha 3 \mu \nu}\right] \frac{\partial}{\partial x_{\nu}}$.

Define then a surface response operator $\overleftrightarrow{A}$ such that its elements are

$$
A_{\alpha \gamma}\left(\mathbf{x}, \mathbf{x}^{\prime}\right)=\int \sum_{\nu} V_{\alpha \mu}(\mathbf{x}) G_{\mu \gamma}\left(\mathbf{x}, \mathbf{x}^{\prime}\right) \mathrm{d} \mathbf{x}
$$

where the bulk response function $\vec{G}$ is defined by

$$
\sum_{\mu} H_{\alpha \mu}(\mathbf{x}) G_{\mu \nu}\left(\mathbf{x}, \mathbf{x}^{\prime}\right)=\delta_{\alpha \nu} \delta\left(\mathbf{x}-\mathbf{x}^{\prime}\right) .
$$

Knowing the bulk response function $\overleftrightarrow{G}$ and the surface response operator $\overleftrightarrow{A}$, one can obtain the response function $\vec{g}_{\mathrm{s}}$ of the homogeneous elastic material bounded by the free surface defined by equation (2.5) from the general equation [5]

$$
\overleftrightarrow{g}_{\mathrm{s}}(\overleftrightarrow{I}+\overleftrightarrow{A})=\overleftrightarrow{G}
$$

where $\vec{I}$ is the identity matrix.

It is useful to use the following notation: D for the space in which the elastic matter is defined and $M$ for the space of its free surface. Then equation (2.13) provides [5]

$$
\overleftrightarrow{\mathrm{g}}_{\mathrm{s}}(\mathrm{DD})=\overleftrightarrow{G}(\mathrm{DD})-\overleftrightarrow{G}(\mathrm{DM}) \overleftrightarrow{\Delta}^{-1}(\mathrm{MM}) \overleftrightarrow{A}(\mathrm{MD}) \text {, }
$$

where $\vec{\Delta}^{-1}(\mathrm{MM})$ is the inverse matrix of

$$
\Delta(\mathrm{MM})=\overleftrightarrow{I}(\mathrm{MM})+\overleftrightarrow{A}(\mathrm{MM}) .
$$

A useful particular value of equation (2.14) is

$$
\stackrel{\leftrightarrow}{\mathrm{s}}_{\mathrm{s}}^{-1}(\mathrm{MM})=\overleftrightarrow{\Delta}(\mathrm{MM}) \stackrel{\leftrightarrow}{G}^{-1}(\mathrm{MM}),
$$

where $\ddot{g}_{\mathrm{s}}^{-1}(\mathrm{MM})$ and $\overleftrightarrow{G}^{-1}(\mathrm{MM})$ are the inverse matrices of $\stackrel{\mathrm{g}}{\mathrm{s}}_{\mathrm{s}}(\mathrm{MM})$ and $\overleftrightarrow{G}(\mathrm{MM})$, respectively.

Let us stress that in order to calculate the elements of $\vec{g}_{\mathrm{s}}$ from equations (2.14) and (2.16), one has in general to discretize the surface space $M$, in order to calculate the inverse matrices defined above.

2.2 The general EQUATIONS FOR A COMPOSITE MATERIAL. - Let us now consider any composite material contained in its space of definition $\mathrm{D}$ and formed out of $N$ different homogeneous pieces situated in their spaces $\mathrm{D}_{i}$. Each piece is bounded by an interface $\mathbf{M}_{i}$, adjacent in general to $j(1 \leqslant j \leqslant J)$ other pieces through subinterface domains $\mathbf{M}_{i j}$. The ensemble of all these interface spaces $\mathbf{M}_{i}$ will be called the interface space $\mathrm{M}$ of the composite material. 
Considering first each piece with its free surface, one can calculate from equation (2.16) its

$$
\ddot{g}_{\mathrm{s}}^{-1}\left(\mathrm{M}_{i} \mathrm{M}_{i}\right)=\overleftrightarrow{\Delta}\left(\mathrm{M}_{i} \mathrm{M}_{i}\right) \overleftrightarrow{G}^{-1}\left(\mathrm{M}_{i} \mathrm{M}_{i}\right)
$$

Then the corresponding $\vec{g}^{-1}(\mathrm{MM})$ for the composite material is obtained [5] in the following manner

$$
\begin{aligned}
& \ddot{\boldsymbol{g}}^{-1}\left(\mathbf{M}_{i j} \mathbf{M}_{i^{\prime} j^{\prime}}\right)=\overrightarrow{0}, \quad \mathbf{M}_{i^{\prime} j^{\prime}} \notin \mathbf{M}_{i} \\
& \ddot{g}^{-1}\left(\mathbf{M}_{i j} \mathbf{M}_{i j^{\prime}}\right)=\ddot{g}_{\mathrm{s}}^{-1}\left(\mathbf{M}_{i j} \mathbf{M}_{i j^{\prime}}\right), \quad j^{\prime} \neq j ; \\
& \overleftrightarrow{\mathrm{g}}^{-1}\left(\mathbf{M}_{i j} \mathbf{M}_{i j}\right)=\sum_{i^{\prime}} \vec{g}_{\mathrm{s}}^{-1}\left(\mathbf{M}_{i^{\prime} j^{\prime}} \mathbf{M}_{i^{\prime} j^{\prime}}\right), \quad \mathbf{M}_{i^{\prime} j^{\prime}} \equiv \mathbf{M}_{i j} \text {. }
\end{aligned}
$$

All the boundary conditions at the interfaces are satisfied through these equations (2.18).

By taking a discrete number of points in the total interface space, one calculates then the inverse $\ddot{g}(\mathrm{MM})$ of $\ddot{g}^{-1}(\mathrm{MM})$. Let us define here $\vec{G}(\mathrm{DD})$ as a block diagonal reference response function constructed out ot the elements of the bulk response functions $\overleftrightarrow{G}\left(\mathrm{D}_{i} \mathrm{D}_{i}\right)$. from

All the elements of the response function $\ddot{g}$ of the composite material can be obtained [5]

$$
\begin{aligned}
& \overleftrightarrow{g}(\mathrm{DD})=\overleftrightarrow{G}(\mathrm{DD})-\overleftrightarrow{G}(\mathrm{DM}) \overleftrightarrow{G}^{-1}(\mathrm{MM}) \overleftrightarrow{G}(\mathrm{MD})+ \\
& +\overleftrightarrow{G}(\mathrm{DM}) \overleftrightarrow{G}^{-1}(\mathrm{MM}) \overleftrightarrow{g}(\mathrm{MM}) \overleftrightarrow{G}^{-1}(\mathrm{MM}) \overleftrightarrow{G}(\mathrm{MD}) \text {. }
\end{aligned}
$$

The new interface states can be calculated from

$$
\operatorname{det}\left|\vec{g}^{-1}(\mathrm{MM})\right|=0
$$

and the total variation of the density of states $\Delta N\left(\omega^{2}\right)$ between the composite material and the reference one was shown [5] to be

$$
\Delta N\left(\omega^{2}\right)=\frac{1}{\pi} \frac{\mathrm{d} \eta\left(\omega^{2}\right)}{\mathrm{d} \omega^{2}}+\frac{1}{\pi} \sum_{i=1}^{N} \operatorname{Im} \operatorname{Tr}\left[\ddot{G}\left(\mathrm{D}_{i} \mathrm{M}_{i}\right) \ddot{G}^{-1}\left(\mathrm{M}_{i} \mathrm{M}_{i}\right) \ddot{G}\left(\mathrm{M}_{i} \mathrm{D}_{i}\right)\right],
$$

where the phase shift $\eta\left(\omega^{2}\right)$ is given by

$$
\eta\left(\omega^{2}\right)=-\arg \operatorname{det}\left|\vec{g}^{-1}(\mathrm{MM})\right| .
$$

For perturbation defects, the equation (2.21) reduces to that first proposed in 1952 by Lifshitz for phonons around point defects and by Friedel [1] for free electrons around impurities in metals and used by him to derive the well known Friedel sum rule. The results (2.18-2.22) in slightly different forms were obtained also, within the Surface Green's Function Matching Theory [8] for materials with one, two and two periodic interfaces. The generalization to any composite material was achieved afterwards within the Interface Response Theory [5].

Let us stress finally that if $|U(\mathrm{D})\rangle$ represents an eigenvector of the reference system, equation (2.19) enables one to calculate the eigenvectors $|u(\mathrm{D})\rangle$ of the composite material [9]

$$
\begin{aligned}
& |u(\mathrm{D})\rangle=|U(\mathrm{D})\rangle-\overleftrightarrow{G}(\mathrm{DM}) \vec{G}^{-1}(\mathrm{MM})|U(\mathrm{M})\rangle+
\end{aligned}
$$

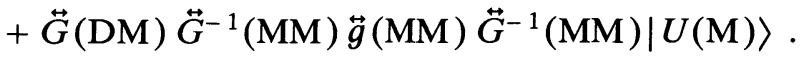


In particular if $|U(\mathrm{D})\rangle$ represents a bulk wave launched in one homogeneous piece of the composite material, equation (2.23) enables to calculate all the waves reflected and transmitted by the interfaces.

Rather than expanding more on these general results, let us illustrate them by a few applications to layered composite elastic materials.

\section{Layered composite materials.}

Any layered composite material can be built out of semi-infinite and slab pieces. We will therefore first show here how to obtain from equation (2.17) the corresponding $\overleftrightarrow{g}_{\mathrm{s}}^{-1}\left(\mathrm{M}_{i} \mathrm{M}_{i}\right)$ for a semi-infinite solid and then for a slab. Then we will indicate how these results can be also used for viscous and non viscous fluids. Finally we will describe how to use these results for any layered composite.

In all what follows we assume the interfaces perpendicular to the $x_{3}$ axis. Then the function $f\left(x_{1}, x_{2}\right)$ vanishes (see Eq. (2.5)). Taking advantage of the infinitesimal translational invariance of this layered composite in directions parallel to the interfaces, one can Fourier analyze all operators, and in particular the response functions $\overleftrightarrow{g}$ and $\vec{G}$, according to

$$
G_{\alpha \beta}\left(\mathbf{x}, \mathbf{x}^{\prime}\right)=\int \frac{\mathrm{d}^{2} \mathbf{k}_{\|}}{(2 \pi)^{2}} g_{\alpha \beta}\left(\mathbf{k}_{\|} \mid x_{3} x_{3}^{\prime}\right) \mathrm{e}^{i \mathbf{k}_{\mathbf{y}} \cdot\left(\mathbf{x}_{\mathbf{q}}-\mathbf{x} \mid\right)}
$$

where

$$
\mathbf{k}_{\|}=\hat{\mathbf{i}}_{1} k_{1}+\hat{\mathbf{i}}_{2} k_{2}
$$

and

$$
\mathbf{x}_{\|}=\hat{\mathbf{i}}_{1} x_{1}+\hat{\mathbf{i}}_{2} x_{2},
$$

$\hat{\mathbf{i}}_{1}$ and $\hat{\mathbf{i}}_{2}$ being unit vectors in the 1- and 2-directions, respectively. Using equations (2.8a) and (2.12), one finds that the Fourier coefficient $G_{\alpha \beta}\left(k_{\mid} \mid x_{3} x_{3}^{\prime}\right)$ of the bulk response function $\vec{G}$ is the solution of the following system of ordinary differential equations

$$
\begin{aligned}
\sum_{\mu}\left\{\delta_{\alpha \mu} \rho \omega^{2}+\sum_{\beta \nu}\right. & C_{\alpha \beta \mu \nu}\left[\left(1-\delta_{\beta 3}\right) i k_{\beta}+\delta_{\beta 3} \frac{\mathrm{d}}{\mathrm{d} x_{3}}\right] \times \\
\times & {\left.\left[\left(1-\delta_{\nu 3}\right) i k_{\nu}+\delta_{\nu 3} \frac{\mathrm{d}}{\mathrm{d} x_{3}}\right]\right\} \cdot G_{\mu \gamma}\left(\mathbf{k}_{\|} \mid x_{3} x_{3}^{\prime}\right)=\delta_{\alpha \gamma} \delta\left(\mathbf{x}_{3}-\mathbf{x}_{3}^{\prime}\right) . }
\end{aligned}
$$

Let us now choose an isotropic elastic medium for which the bulk response function $\overleftrightarrow{G}$ can be calculated in closed form.

3.1 BULK RESPONSE FUNCTION FOR AN ISOTROPIC ELASTIC SOLID. - An isotropic elastic medium is a medium whose properties are isotropic in all directions of space. For such a medium

$$
C_{\alpha \beta \mu \nu}=c_{12} \delta_{\alpha \beta} \delta_{\mu \nu}+c_{44}\left(\delta_{\alpha \mu} \delta_{\beta \nu}+\delta_{\alpha \nu} \delta_{\beta \mu}\right)
$$

with

$$
c_{12}=c_{11}-2 c_{44} \text {. }
$$


The square of the longitudinal and shear plane wave velocities are respectively

$$
c_{1}^{2}=\frac{c_{11}}{\rho} \quad \text { and } \quad c_{\mathrm{t}}^{2}=\frac{c_{44}}{\rho} .
$$

Such a material is also isotropic within the $\left(x_{1}, x_{2}\right)$ plane. It is possible to choose in equations (3.2) and (3.4) $k_{2}=0$. The results obtained for $\vec{G}\left(k_{1} \mid x_{3} x_{3}^{\prime}\right)$ can be used for any other direction of $\mathbf{k}_{\|}$, after a rotation of the $\mathbf{x}_{1}$ and $\mathbf{x}_{2}$ axes such that $\mathbf{k}_{\|}$lies along $\mathbf{x}_{1}$. As in this case $\left|\mathbf{k}_{\|}\right|=k_{\|}=k_{1}$, we will write them as function of $k_{\|}$rather than $k_{1}$.

Let us define also

$$
\begin{aligned}
& \alpha_{1}^{2}=k_{\|}^{2}-\frac{\omega^{2}}{c_{1}^{2}}, \\
& \alpha_{\mathrm{t}}^{2}=k_{\|}^{2}-\frac{\omega^{2}}{c_{\mathrm{t}}^{2}},
\end{aligned}
$$

and

$$
\varepsilon=\frac{\alpha_{\mathrm{t}} \alpha_{1}}{k_{\|}^{2}}
$$

Another interesting property due to the isotropy in the $\left(x_{1}, x_{2}\right)$ plane is the decoupling of the transverse vibrations polarized along $x_{2}$ from the sagittal vibrations polarized in the $\left(x_{1}, x_{3}\right)$ plane. This decoupling will remain for all layered composites and it is possible to study separately the $x_{2}$ polarized transverse vibrations and the sagittal ones in such composites.

The elements of $\overleftrightarrow{G}\left(k_{\|} \omega \mid x_{3} x_{3}^{\prime}\right)$ are [10]

$$
\begin{aligned}
& G_{\alpha 2}\left(k_{\|} \mid x_{3} x_{3}^{\prime}\right)=G_{2 \alpha}\left(k_{\|} \omega \mid x_{3} x_{3}^{\prime}\right)=0, \quad \alpha=1,3, \\
& G_{11}\left(k_{\|} \mid x_{3} x_{3}^{\prime}\right)=-\frac{k_{\|}^{2}}{2 \rho \alpha_{1} \omega^{2}}\left[\mathrm{e}^{-\alpha_{1}\left|x_{3}-x_{3}^{\prime}\right|}-\varepsilon \mathrm{e}^{-\alpha_{\mathrm{t}}\left|x_{3}-x_{3}^{\prime}\right|}\right], \\
& G_{13}\left(k_{\|} \mid x_{3} x_{3}^{\prime}\right)=-\frac{i k_{\|}}{2 \rho \omega^{2}} \operatorname{sgn}\left(x_{3}-x_{3}^{\prime}\right)\left[\mathrm{e}^{-\alpha_{\mathrm{t}}\left|x_{3}-x_{3}^{\prime}\right|}-\mathrm{e}^{-\alpha_{1}\left|x_{3}-x_{3}^{\prime}\right|}\right], \\
& \mathrm{G}_{22}\left(k_{\|} \mid x_{3} x_{3}^{\prime}\right)=\frac{-1}{2 \rho \alpha_{\mathrm{t}} c_{\mathrm{t}}^{2}} \mathrm{e}^{-\alpha_{\mathrm{t}}\left|x_{3}-x_{3}^{\prime}\right|}, \\
& G_{31}\left(k_{\|} \mid x_{3} x_{3}^{\prime}\right)=-\frac{i k_{\|}}{2 \rho \omega^{2}} \operatorname{sgn}\left(x_{3}-x_{3}^{\prime}\right)\left[\mathrm{e}^{-\alpha_{\mathrm{t}}\left|x_{3}-x_{3}^{\prime}\right|}-\mathrm{e}^{-\alpha_{1}\left|x_{3}-x_{3}^{\prime}\right|}\right], \\
& G_{33}\left(k_{\|} \mid x_{3} x_{3}^{\prime}\right)=\frac{-k_{\|}^{2}}{2 \rho \alpha_{\mathrm{t}} \omega^{2}}\left[-\varepsilon \mathrm{e}^{-\alpha_{1}\left|x_{3}-x_{3}^{\prime}\right|}+\mathrm{e}^{-\alpha_{\mathrm{t}}\left|x_{3}-x_{3}^{\prime}\right|}\right] .
\end{aligned}
$$

Let us remark that $\alpha_{\mathrm{t}}=\left(k_{\|}^{2}-\omega^{2} / c_{\mathrm{t}}^{2}\right)^{1 / 2}$ is real for $\omega<c_{\mathrm{t}} k_{\|}$and that we choose $\alpha_{\mathrm{t}}=-i\left(\omega^{2} / c_{\mathrm{t}}^{2}-k_{\|}^{2}\right)^{1 / 2}$ for $\omega>c_{\mathrm{t}} k_{\|}$. The negative sign in this last result corresponds in the response functions to outgoing waves at $x_{3}= \pm \infty$. The same consideration applies to $\alpha_{1}$. 
3.2 ONE SEMI-INFINITE SOLID. - Let us consider now a semi-infinite solid such that $x_{3} \geqslant 0$. For the isotropic solid described above and for $\mathbf{k}_{\|}=\hat{\mathbf{i}} k_{\|}$, the elements of the cleavage operator defined by equation (2.10) become

$$
\begin{aligned}
& V_{\alpha 2}\left(k_{\|} \mid x_{3}\right)=V_{2 \alpha}\left(k_{\|} \mid x_{3}\right)=0, \quad \alpha=1,3, \\
& V_{11}\left(k_{\|} \mid x_{3}\right)=c_{44} \delta\left(x_{3}\right) \frac{\mathrm{d}}{\mathrm{d} x_{3}}, \\
& V_{13}\left(k_{\|} \mid x_{3}\right)=i k_{\|} c_{44} \delta\left(x_{3}\right), \\
& V_{22}\left(k_{\|} \mid x_{3}\right)=c_{44} \delta\left(x_{3}\right) \frac{\mathrm{d}}{\mathrm{d} x_{3}}, \\
& V_{31}\left(k_{\|} \mid x_{3}\right)=i k_{\|} c_{12} \delta\left(x_{3}\right), \\
& V_{33}\left(k_{\|} \mid x_{3}\right)=c_{11} \delta\left(x_{3}\right) \frac{\mathrm{d}}{\mathrm{d} x_{3}} .
\end{aligned}
$$

With the help of equations (2.11), (2.15), (2.16) and (3.12) one obtains

$$
\ddot{g}_{\mathrm{s}}^{-1}(00)=\left[\begin{array}{ccc}
-\frac{\rho \omega^{2} \alpha_{1}}{k_{\|}^{2}(1-\varepsilon)} & 0 & -i \rho k_{\|}\left[-2 c_{\mathrm{t}}^{2}+\frac{\omega^{2}}{k_{\|}^{2}(1-\varepsilon)}\right] \\
0 & -\rho \alpha_{\mathrm{t}} c_{\mathrm{t}}^{2} & 0 \\
+i \rho k_{\|}\left[-2 c_{\mathrm{t}}^{2}+\frac{\omega^{2}}{k_{\|}^{2}(1-\varepsilon)}\right] & 0 & -\frac{\rho \omega^{2} \alpha_{\mathrm{t}}}{k_{\|}^{2}(1-\varepsilon)}
\end{array}\right] .
$$

Let us note that the $\ddot{g}_{\mathrm{s}}^{-1}(00)$ for the same elastic medium but such that $x_{3} \leqslant 0$ has the same expression (3.14) but with a change of sign in the off-diagonal elements coupling the $x_{1}$ and $x_{3}$ polarisations.

Note also that

$$
\operatorname{det}\left|g_{\mathrm{s}}^{-1}(00)\right|=-\frac{\rho^{2} c_{\mathrm{t}}^{4}}{k_{\|}^{2}(1-\varepsilon)}\left[\left(\alpha_{\mathrm{t}}^{2}+k_{\|}^{2}\right)^{2}-4 k_{\|}^{2} \alpha_{\mathrm{t}} \alpha_{1}\right],
$$

from which, with the help of equation (2.20) one obtains the well known Rayleigh wave dispersion relation.

With the help of equation (2.14), one also recovers [10] the response function $\vec{g}_{\mathrm{s}}$ of the semi-infinite solid.

3.3 A FREE SURFACE SOLID SLAB. - Let us now consider an isotropic elastic slab such that

$$
-a \leqslant x_{3} \leqslant a .
$$

The interface space $M$ is now formed out of these two surfaces $x_{3}= \pm a$. The cleavage operator has contributions proportional to $\delta\left(x_{3}+a\right)$ and to $\delta\left(x_{3}-a\right)$; the coefficients of the first one are equal to those given by equation (3.13), the signs of the coefficients of the second contribution are just changed. As above, one can calculate the corresponding $\vec{g}_{\mathrm{s}}^{-1}(\mathrm{MM})$. Since the transverse vibrations polarized along $\mathbf{x}_{2}$ decouple from the sagittal ones, it is convenient to derive their contribution to this $\overleftrightarrow{\mathrm{g}}_{\mathrm{s}}^{-1}(\mathrm{MM})$ separately namely

$$
\ddot{g}_{\mathrm{s} 22}^{-1}(\mathrm{MM})=-\frac{\rho c_{\mathrm{t}}^{2} \alpha_{\mathrm{t}}}{\operatorname{sh} 2 \alpha_{\mathrm{t}} a}\left[\begin{array}{cc}
\operatorname{ch} 2 \alpha_{\mathrm{t}} a & -1 \\
-1 & \operatorname{ch~} 2 \alpha_{\mathrm{t}} a
\end{array}\right]
$$


The calculation of the sagittal contribution to $\vec{g}_{\mathrm{s}}^{-1}(\mathrm{MM})$ can be obtained more easily, with the help of the reflection symmetry through the middle of the slab. This decouples the corresponding $4 \times 4$ matrix in two $(2 \times 2)$ matrices : $\vec{g}_{\mathrm{ss}}^{-1}(\mathrm{MM})$ for the symmetrical coordinates

$$
\left\{\frac{\left|-a, x_{1}\right\rangle+\left|a, x_{1}\right\rangle}{\sqrt{2}}, \frac{\left|-a, x_{3}\right\rangle-\left|a, x_{3}\right\rangle}{\sqrt{2}}\right\}
$$

and $\overleftrightarrow{g}_{\mathrm{sAs}}^{-1}$ for the antisymmetrical coordinates

$$
\left\{\frac{\left|-a, x_{1}\right\rangle-\left|-a, x_{1}\right\rangle}{\sqrt{2}}, \frac{\left|-a, x_{3}\right\rangle+\left|a, x_{3}\right\rangle}{\sqrt{2}}\right\}
$$

respectively. Their expressions are

where

$$
\stackrel{\mathrm{g}}{\mathrm{ss}}_{\mathrm{is}}^{-1}(\mathrm{MM})=\frac{1}{d_{\mathrm{s}}}\left[\begin{array}{cc}
\frac{\alpha_{1 \omega^{2}}}{k_{\|} c_{\mathrm{t}}^{2}} \operatorname{sh} \alpha_{1} a \operatorname{sh} \alpha_{\mathrm{t}} a & i\left[2 \alpha_{1} \alpha_{\mathrm{t}} \operatorname{ch} \alpha_{\mathrm{t}} a \operatorname{sh} \alpha_{1} a-\right. \\
-i\left[2 \alpha_{1} \alpha_{\mathrm{t}} \operatorname{ch} \alpha_{\mathrm{t}} a \operatorname{sh} \alpha_{1} a\right. & \left.\left(\alpha_{\mathrm{t}}^{2}+k_{\|}^{2}\right) \operatorname{sh} \alpha_{\mathrm{t}} a \operatorname{ch} \alpha_{1} a\right] \\
\left.-\left(\alpha_{\mathrm{t}}^{2}+k_{\|}^{2}\right) \operatorname{ch} \alpha_{1} a \operatorname{sh} \alpha_{\mathrm{t}} a\right] & \frac{\alpha_{\mathrm{t}} \frac{\omega^{2}}{k_{\|}} \operatorname{ch} \alpha_{1} a \operatorname{ch} \alpha_{\mathrm{t}} a}{c_{\mathrm{t}}^{2}}
\end{array}\right]
$$

$$
d_{\mathrm{s}}=-\frac{k_{\|}}{\rho c_{\mathrm{t}}^{2}}\left[\operatorname{ch} \alpha_{1} a \operatorname{sh} \alpha_{\mathrm{t}} a-\varepsilon \operatorname{ch} \alpha_{\mathrm{t}} a \operatorname{sh} \alpha_{1} a\right]
$$

and

where

$$
\ddot{g}_{\mathrm{SAs}}^{-1}(\mathrm{MM})=\frac{1}{d_{\mathrm{As}}}\left[\begin{array}{cc}
\frac{\alpha_{1}}{k_{\|} \frac{\omega^{2}}{c_{\mathrm{t}}^{2}} \operatorname{ch} \alpha_{1} a \operatorname{ch} \alpha_{\mathrm{t}} a} & i\left[2 \alpha_{1} \alpha_{\mathrm{t}} \operatorname{sh} \alpha_{\mathrm{t}} a \operatorname{ch} \alpha_{1} a-\right. \\
-i\left[2 \alpha_{1} \alpha_{\mathrm{t}} \operatorname{sh} \alpha_{\mathrm{t}} a \operatorname{ch} \alpha_{1} a\right. & \left.\left(\alpha_{\mathrm{t}}^{2}+k_{\|}^{2}\right) \operatorname{ch} \alpha_{\mathrm{t}} a \operatorname{sh} \alpha_{1} a\right] \\
\left.-\left(\alpha_{\mathrm{t}}^{2}+k_{\|}^{2}\right) \operatorname{sh} \alpha_{1} a \operatorname{ch} \alpha_{\mathrm{t}} a\right] & \frac{\alpha_{\mathrm{t}} \frac{\omega^{2}}{k_{\|}} \operatorname{sh} \alpha_{1} a \operatorname{sh} \alpha_{\mathrm{t}} a}{c_{\mathrm{t}}^{2}}
\end{array}\right]
$$

$$
d_{\mathrm{As}}=-\frac{k_{\|}}{\rho c_{\mathrm{t}}^{2}}\left[\operatorname{sh} \alpha_{1} a \operatorname{ch} \alpha_{\mathrm{t}} a-\varepsilon \operatorname{sh} \alpha_{\mathrm{t}} a \operatorname{ch} \alpha_{1} a\right]
$$

The above expressions are particularly useful for composites keeping this reflexion symmetry through the middle of a central slab. In general, however, we will need the $(4 \times 4)$ expression of the slab $\overleftrightarrow{g}_{s}^{-1}(\mathrm{MM})$ for the normal coordinates $\left\{\left|-a, x_{1}\right\rangle,\left|-a, x_{3}\right\rangle\right.$, $\left.\left|a, x_{1}\right\rangle,\left|a, x_{3}\right\rangle\right\}$. This expression is easily obtained from equation (3.18) to be

$$
\ddot{\boldsymbol{g}}_{\mathrm{s}}^{-1}(\mathrm{MM})=\left[\begin{array}{cccc}
a_{0} & i q & d & i f \\
-i q & b & i f & e \\
d & -i f & a_{0} & -i q \\
-i f & e & i q & b
\end{array}\right]
$$


where

$F=-\frac{\rho c_{\mathrm{t}}^{2}}{2 k_{\|}}\left(\operatorname{ch} \alpha_{1} a \operatorname{sh} \alpha_{\mathrm{t}} a-\varepsilon \operatorname{ch} \alpha_{\mathrm{t}} a \operatorname{sh} \alpha_{1} a\right)^{-1}\left(\operatorname{sh} \alpha_{1} a \operatorname{ch} \alpha_{\mathrm{t}} a-\varepsilon \operatorname{sh} \alpha_{\mathrm{t}} a \operatorname{ch} \alpha_{1} a\right)^{-1}$,

$a_{0}=\frac{F \alpha_{1} \omega^{2}}{2 k_{\|} c_{\mathrm{t}}^{2}}\left[\operatorname{sh} 2 \alpha_{\mathrm{t}} a \operatorname{ch} 2 \alpha_{1} a-\varepsilon \operatorname{sh} 2 \alpha_{1} a \operatorname{ch} 2 \alpha_{\mathrm{t}} a\right]$,

$b=\frac{F \alpha_{\mathrm{t}} \omega^{2}}{2 k_{\|} c_{\mathrm{t}}^{2}}\left[\operatorname{sh} 2 \alpha_{1} a \operatorname{ch} 2 \alpha_{\mathrm{t}} a-\varepsilon \operatorname{sh} 2 \alpha_{\mathrm{t}} a \operatorname{ch} 2 \alpha_{1} a\right]$,

$q=F\left\{\varepsilon\left(3 k_{\|}^{2}+\alpha_{\mathrm{t}}^{2}\right)\left(\operatorname{sh}^{2} \alpha_{1} a \operatorname{ch}^{2} \alpha_{\mathrm{t}} a+\operatorname{sh}^{2} \alpha_{\mathrm{t}} a \operatorname{ch}^{2} \alpha_{1} a\right)-\right.$ $\left.-\frac{1}{2}\left[2 \alpha_{1} \alpha_{\mathrm{t}} \varepsilon+\left(\alpha_{\mathrm{t}}^{2}+k_{\|}^{2}\right)\right] \operatorname{sh} 2 \alpha_{\mathrm{t}} a \operatorname{sh} 2 \alpha_{1} a\right\}$,

$d=-\frac{F \alpha_{1} \omega^{2}}{2 k_{\|} c_{\mathrm{t}}^{2}}\left(\operatorname{sh} 2 \alpha_{\mathrm{t}} a-\varepsilon \operatorname{sh} 2 \alpha_{1} a\right)$,

$e=-\frac{F \alpha_{\mathrm{t}} \omega^{2}}{2 k_{\|} c_{\mathrm{t}}^{2}}\left(\operatorname{sh} 2 \alpha_{1} a-\varepsilon \operatorname{sh} 2 \alpha_{\mathrm{t}} a\right)$,

$f=-F\left[2 \alpha_{1} \alpha_{\mathrm{t}}-\varepsilon\left(\alpha_{\mathrm{t}}^{2}+k_{\|}^{2}\right)\right]\left(\mathrm{sh}^{2} \alpha_{1} a-\operatorname{sh}^{2} \alpha_{\mathrm{t}} a\right)$.

3.4 THE SPECIAL CASE OF FLUIDS. - It was shown before [11] that the motion of a fluid governed by the linearized Navier-Stokes equation can be studied with the help of the equations that describe the motion of a solid providing that

$$
c_{1}^{2}=c_{0}^{2}-\frac{i \omega}{\rho_{0}}\left(\mu^{\prime}+\frac{4}{3} \mu\right)
$$

and

$$
c_{\mathrm{t}}^{2}=-\frac{i \omega \mu}{\rho_{0}}
$$

where $c_{0}$ is the longitudinal speed of sound in the fluid, $\rho_{0}$ its density, and $\mu$ and $\mu^{\prime}$ are the coefficients of shear and dilatation viscosity.

A non-viscous fluid can also be studied from the above equations, by taking the limit $c_{\mathrm{t}} \rightarrow 0$ in them.

3.5 ANY LAYERED ELASTIC COMPOSITE. - We have now all the ingredients necessary for the study of the elastic properties of any layered composite with fluid and solid parts. Once the $\vec{g}_{s}^{-1}\left(M_{i} M_{i}\right)$ for each different part is determined, then the $\ddot{g}^{-1}(M M)$ of the composite is obtained by linear superposition of these $\vec{g}_{\mathrm{s}}^{-1}\left(\mathrm{M}_{i} \mathrm{M}_{i}\right)$, as specified by equations (2.18). Then the main basic properties of the composite can be obtained with the help of equations (2.19)(2.23). Rather than expanding these general results, let us illustrate them by an application to the vibrations of a fluid and a solid slab sandwiched between two semi-infinite solids.

\section{A few applications.}

4.1 THE FLUID SANDWICH VIBRATIONS. - Let us consider a non-viscous fluid slab of thickness $2 a$ sandwiched between two identical semi-infinite solids. The application of the theory to this composite easily provides closed form expressions for the sandwich vibrations 
localized within the fluid slab. Note first that there are no transverse polarized modes in the fluid slab.

The expression giving the symmetrical sagittal sandwich modes was found to be

$$
\left(\alpha_{\mathrm{t}}^{2}+k_{\|}^{2}\right)^{2}-4 k_{\|}^{2} \alpha_{\mathrm{t}} \alpha_{1}+\frac{\rho_{0}}{\rho} \frac{\omega^{4}}{c_{\mathrm{t}}^{4}} \frac{\alpha_{1}}{\alpha_{0}} \operatorname{coth} \alpha_{0} a=0
$$

and those for the antisymmetrical sagittal ones

$$
\left(\alpha_{\mathrm{t}}^{2}+k_{\|}^{2}\right)^{2}-4 k_{\|}^{2} \alpha_{\mathrm{t}} \alpha_{1}+\frac{\rho_{0}}{\rho} \frac{\omega^{4}}{c_{\mathrm{t}}^{4}} \frac{\alpha_{1}}{\alpha_{0}} \text { th } \alpha_{0} a=0,
$$

where the $\rho_{0}$ and $\rho$ are the mass densities of the fluid and of the solid respectively; $\alpha_{1}$ and $\alpha_{\mathrm{t}}$ are the outgoing at infinity penetration coefficients of the solid given by equations (3.8)-(3.9) and $\alpha_{0}$ is the equivalent of $\alpha_{1}$ for the longitudinal wave in the liquid.

Remark that in the limit $a \rightarrow \infty$, coth $\alpha_{0} a$ and th $\alpha_{0} a \rightarrow 1$, both equations (4.1) and (4.2) reduce to the expression for the localized vibrations at a planar fluid-solid interface [12].

Figure 1 represents the first symmetrical and anti-symmetrical modes localized within the fluid slab. Defining the speed of these modes by $c$, we represented $c / c_{t}=f\left(k_{\|} a\right)$ for the parameters characteristic of an acoustic Perot-Fabry interferometer [13]. In connexion with this experiment, using equations (2.23) we also calculated the reflected and transmitted waves due to an acoustic wave launched in the solid. These results are given in the appendix.

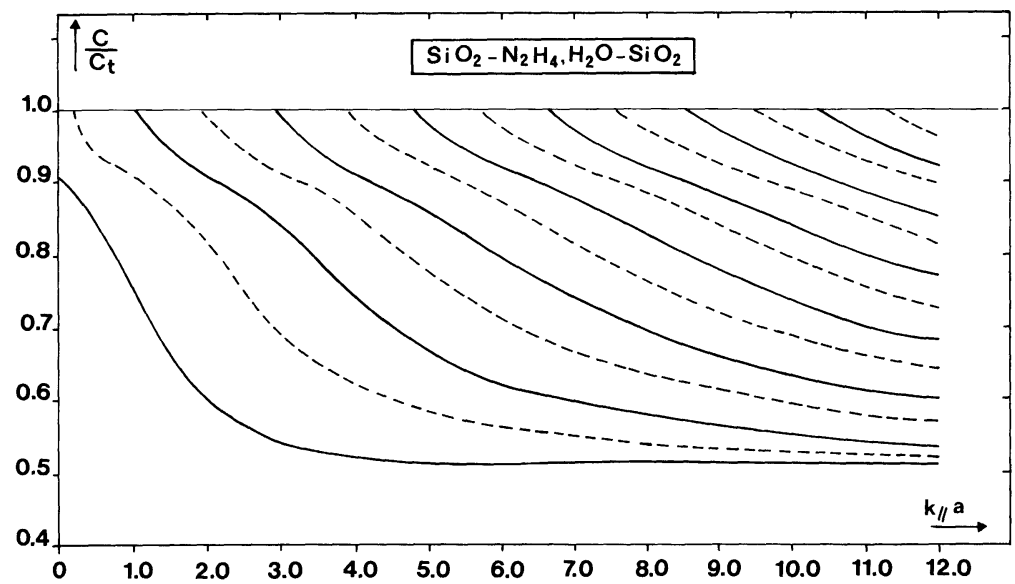

Fig. 1. - Localized waves in a thin fluid slab of thickness $2 a$ sandwiched between two identical semiinfinite solids. The figure displays the ratio of the speed of propagation $c$ of these waves to the transverse speed of sound $c_{\mathrm{t}}$ in the solid as a function of $a k_{\|}$. The parameters used in this calculation are those of an acoustic interferometer, namely : $\rho_{0}=1010 \mathrm{~kg} / \mathrm{m}^{3}, c_{0}=1900 \mathrm{~m} / \mathrm{s}$ for the liquid $\left(\mathrm{N}_{2} \mathrm{H}_{4}, \mathrm{H}_{2} \mathrm{O}\right)$ and $\rho=2200 \mathrm{~kg} / \mathrm{m}^{3}, c_{\mathrm{t}}=3750 \mathrm{~m} / \mathrm{s}$ and $c_{1}=5900 \mathrm{~m} / \mathrm{s}$ for the solid $\left(\mathrm{SiO}_{2}\right)$.

4.2 THE SOLID SANDWICH VIBRATIONS. - Let us now consider a solid slab of thickness $2 a$ sandwiched between two different semi-infinite solids. Using the results (3.14), (3.19) and (2.18), one obtains a $(4 \times 4)$ matrix expression for the sagital part of the $\vec{g}^{-1}(\mathrm{MM})$ associated with this composite and using equations (3.17), (3.14) and (2.18) one obtains a $(2 \times 2)$ matrix for the transverse part. Then equation (2.20) enables us to calculate the localized vibrations within the solid slab. Such localized modes were studied before with another method but only for symmetrical sandwiches [14-15]. 
Let us label the two semi-infinite solids by the subscripts 1 and 2 and keep for the solid slab the same notation without subscript as in section 3.3. The closed form expression giving the localized transverse modes was easily found to be

$$
\rho_{1} c_{\mathrm{t} 1}^{2} \rho_{2} c_{\mathrm{t} 2}^{2} \alpha_{\mathrm{t} 1} \alpha_{\mathrm{t} 2}+\left(\rho_{1} c_{\mathrm{t} 1}^{2} \alpha_{\mathrm{t} 1}+\rho_{2} c_{\mathrm{t} 2}^{2} \alpha_{\mathrm{t} 2}\right) \rho c_{\mathrm{t}}^{2} \alpha_{\mathrm{t}} \operatorname{coth} 2 a \alpha_{\mathrm{t}}+\rho^{2} c_{\mathrm{t}}^{4} \alpha_{\mathrm{t}}^{2}=0
$$

Figure 2 represents the first of the infinity of modes localized within a slab of $\mathrm{W}$ sandwiched between $\mathrm{Ni}$ and $\mathrm{Al}$. The input parameters are given in the table I. The speed $\mathrm{c}$ of these localized modes lies in between the transverse speeds of sound of $\mathrm{Al}$ and $\mathrm{W}$. Note that these localized modes exist only when the material sandwiched between the two others has a transverse speed of sound $c_{\mathrm{t}}$ such that $c_{\mathrm{t}}<c_{\mathrm{t} 1}<c_{\mathrm{t} 2}$. Otherwise one obtains only resonant modes, also called leaky waves [12].

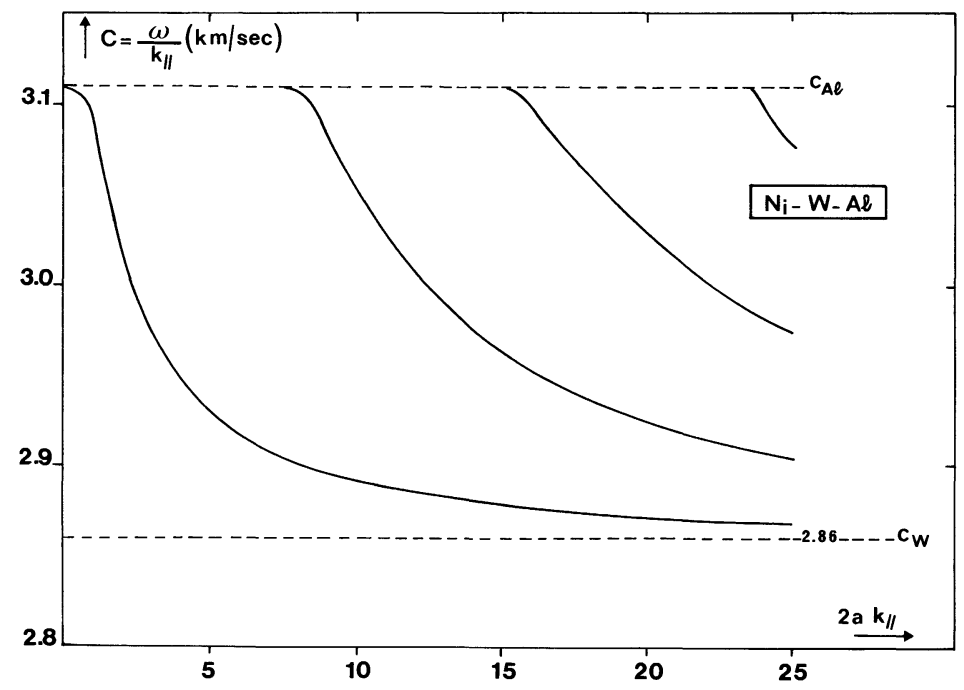

Fig. 2. - Speed $c$ of transverse localized waves in a Ni-W-Al sandwich.

Table I. - Input values for the calculation of the velocities given in figures 2, 3 and 4 .

\begin{tabular}{|c|c|c|c|c|}
\hline & $\begin{array}{c}c_{11} \\
\left(10^{11} \mathrm{~N} / \mathrm{m}^{2}\right)\end{array}$ & $\begin{array}{c}c_{44} \\
\left(10^{11} \mathrm{~N} / \mathrm{m}^{2}\right)\end{array}$ & $\begin{array}{c}c_{1} \\
(\mathrm{~km} / \mathrm{s})\end{array}$ & $\begin{array}{c}c_{\mathrm{t}} \\
(\mathrm{km} / \mathrm{s})\end{array}$ \\
\hline $\mathrm{W}$ & 5.120 & 1.530 & 5.231 & 2.860 \\
$\mathrm{Al}$ & 1.113 & 0.261 & 6.422 & 3.110 \\
$\mathrm{Ni}$ & 3.115 & 0.929 & 5.894 & 3.219 \\
\hline
\end{tabular}

The sagittal modes localized within the sandwich are the solutions of the following equation

$$
\operatorname{det}\left[\begin{array}{cccc}
a_{0}+a_{1} & i\left(q+q_{1}\right) & d & i f \\
-i\left(q+q_{1}\right) & b+b_{1} & i f & e \\
d & -i f & a_{0}+a_{2} & -i\left(q+q_{2}\right) \\
-i f & e & i\left(q+q_{2}\right) & \left(b+b_{2}\right)
\end{array}\right]=0
$$


where the parameters without index are given by equations (3.20) and for $i=1$ and 2

$$
\begin{aligned}
a_{i} & =-\frac{\omega^{2}}{k_{\|}^{2}} \frac{\rho_{i} \alpha_{1_{i}}}{1-\varepsilon_{i}}, \\
b_{i} & =-\frac{\omega^{2}}{k_{\|}^{2}} \frac{\rho_{i} \alpha_{\mathfrak{t}_{i}}}{1-\varepsilon_{i}}, \\
q_{i} & =\rho_{i} k_{\|}\left[-2 c_{t i}^{2}+\frac{\omega^{2}}{k_{\|}^{2}\left(1-\varepsilon_{i}\right)}\right] .
\end{aligned}
$$

In order to discuss the different possible localized sagittal modes in such a sandwich, let us distinguish four cases depending on the respective values of the transverse speeds of sound of the three different materials and on the existence or non-existence of a localized Stoneley wave having velocity $c_{s}$ at a single interface between the central material and one of the others.

i) $c_{\mathrm{s}}<c_{\mathrm{t}}<c_{\mathrm{t} 1}<c_{\mathrm{t} 2}$

An infinity of localized sandwich modes exist. Their speed is $c_{\mathrm{t}}$. The variation of the speed $c$ as a function of $2 a k_{\|}$of the first of these modes is given in figure 3 for the Ni-W-Al sandwich. When $2 a k_{\|} \rightarrow \infty$, the velocity of this sandwich mode goes to the velocity of the Stoneley wave existing at the single W-Al interface. The other modes (not shown in the figure) come out of the bulk band of $\mathrm{Al}$ for higher values of $2 a k_{\|}$and when $2 a k_{\|} \rightarrow \infty$, their velocity tends asymptotically to the velocity $c_{\mathrm{t}}$ of $W$.

ii) $c_{\mathrm{t}}<c_{\mathrm{t} 1}<c_{\mathrm{t} 2}$ with no existence of a Stoneley wave

One expects similar results as above, apart that all localized mode velocities $\mathrm{c}$ will tend to $c_{\mathrm{t}}$ for $2 a k_{\|} \rightarrow \infty$.

iii) $c_{\mathrm{s}}<c_{\mathrm{t} 1}<c_{\mathrm{t}}<c_{\mathrm{t} 2}$

Only one localized mode exists below $c_{\mathrm{t} 1}$ and its velocity $c \rightarrow c_{\mathrm{s}}$ for $2 a k_{\|} \rightarrow \infty$. The velocity of such a mode is represented in figure 4 for a W-Al-Ni sandwich.

iv) $c_{\mathrm{t} 1}<c_{\mathrm{t}}<c_{\mathrm{t} 2}$ and $c_{\mathrm{t} 1}<c_{\mathrm{t} 2}<c_{\mathrm{t}}$

There is no truly localized mode within the sandwich.

\section{Discussion.}

Let us first mention here two other problems which were solved recently with the same formalism.

(i) Closed form expressions for bulk and surface transverse elastic waves in superlattices made of 3 and 4 different layers were obtained [16].

(ii) Analytic expressions for the elastic energy of interaction of a point defect with a planar defect, a plane of dilatation and half a plane of dilatation in a plane defect have been calculated [17]. The implications for segregation of impurities near high angle grain boundaries are also discussed in this work.

Many other problems related in particular to the static and vibrational properties of layered composites can be studied with the present formalism. Let us just mention here those related with the seismic propagation of elastic waves [18], the non-destructive testing of layered multimaterials, their thermodynamic properties, etc. 

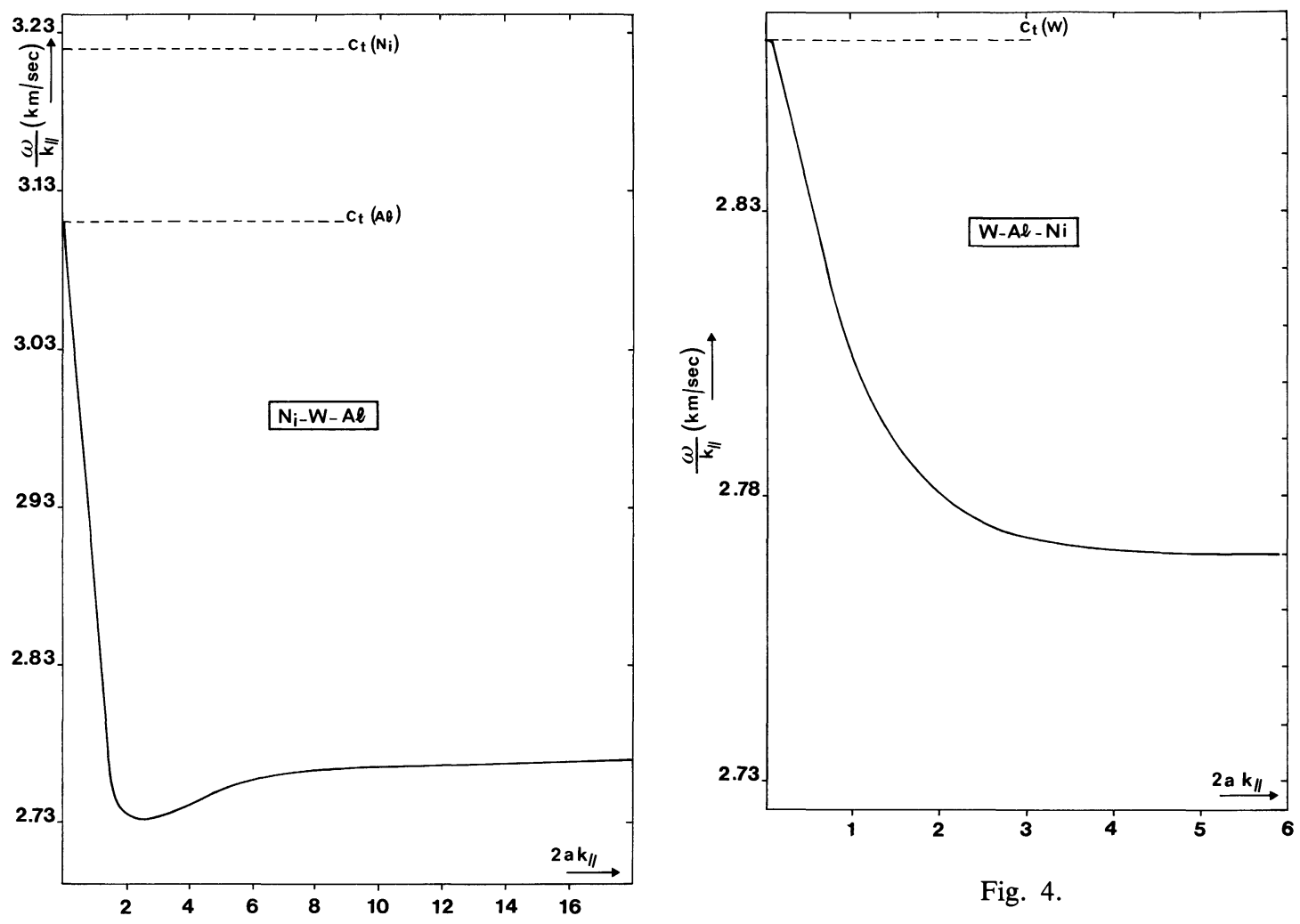

Fig. 4.

Fig. 3.

Fig. 3. - Speed $c$ of the first sagittal localized wave in a Ni-W-Al sandwich.

Fig. 4. - Speed $c$ of the sagittal localized wave in a W-Al-Ni sandwich.

Explicit expressions of the elastic response functions were obtained only for a few layered systems : free surfaces and a planar interface between two static solids [19] with conventional solutions of the differential equations. The formalism described in this paper enables one to consider more complex layered composites mostly because the double boundary conditions (continuity of stresses and displacements at each interface) are combined (see Eqs. (2.18)) into a single boundary condition.

Let us finally stress that the theory outlined in this paper and illustrated by two simple examples of layered composites applies to any elastic composite without any restriction as to the number and the form of the constituents. Of course in such cases and especially if one deals with non-isotropic constituents, the whole theoretical procedure described in Section 2 becomes numerical and should be compared with the finite element method. Advantages were [20] found in the use of the interface response method, mostly because many physical properties require only a calculation within the interface space and because a knowledge of the response function for a given composite avoids having to redo the whole numerical procedure each time one seeks the response of a system (like the S.O.N.A.R. for example) to another excitation. 


\section{Appendix}

The fluid sandwich : localized, reflected and transmitted waves.

Consider a sagittal bulk propagating wave coming from $x_{3}=+\infty$. The corresponding eigenvector has as components

$$
\begin{aligned}
& U_{11}\left(x_{3}\right)=\frac{i k_{\|}}{\alpha_{1}} B_{1} \mathrm{e}^{-\alpha_{1}\left(x_{3}-a\right)}+B_{3} \mathrm{e}^{-\alpha_{\mathrm{t}}\left(x_{3}-a\right)}, \\
& U_{13}\left(x_{3}\right)=B_{1} \mathrm{e}^{-\alpha_{1}\left(x_{3}-a\right)}-\frac{i k_{\|}}{\alpha_{\mathrm{t}}} B_{3} \mathrm{e}^{-\alpha_{\mathrm{t}}\left(x_{3}-a\right)},
\end{aligned}
$$

where

$$
\alpha_{1}=i\left(\frac{\omega^{2}}{c_{1}^{2}}-k_{\|}^{2}\right)^{1 / 2} \text { and } \alpha_{\mathrm{t}}=i\left(\frac{\omega^{2}}{c_{\mathrm{t}}^{2}}-k^{2}\right)^{1 / 2}
$$

Using this incident wave as $|U(\mathrm{D})\rangle$ in equation (2.23), one obtains from the second and third term of this equation the reflected and transmitted waves. Let us recall that the $\alpha_{1}$ and $\alpha_{\mathrm{t}}$ to be used in the response function appearing in this equation were defined as $\alpha_{1}=-i\left(\frac{\omega^{2}}{c_{1}^{2}}-k_{\|}^{2}\right)^{1 / 2}$ for $\omega>k_{\|} c_{1}$ and $\alpha_{\mathrm{t}}=-i\left(\frac{\omega^{2}}{c_{\mathrm{t}}^{2}}-k_{\|}^{2}\right)^{1 / 2}$

$$
\text { for } \omega>k_{\|} c_{\mathrm{t}}
$$

and provide then the reflected and transmitted waves corresponding to the incident wave (A1). In what follows we give the final expressions of these waves, where the $\alpha_{1}$ and $\alpha_{t}$ take the values given by equation (A2). Let us define also

$$
\alpha_{0}= \begin{cases}\left(k_{\|}^{2}-\frac{\omega^{2}}{c_{0}^{2}}\right)^{1 / 2} & \text { for } \omega<k_{\|} c_{0} \\ -i\left(\frac{\omega^{2}}{c_{0}^{2}}-k_{\|}^{2}\right) & \text { for } \omega>k_{\|} c_{0}\end{cases}
$$

and

$$
\begin{gathered}
\hat{B}=\frac{\rho^{2} k_{\|}}{(1-\varepsilon)}\left[\frac{i k_{\|}}{\alpha_{1}}\left(\frac{\omega^{2}}{k_{\|}^{2}}-2 c_{\mathrm{t}}^{2}\right) B_{1}-2 c_{\mathrm{t}}^{2} B_{3}\right] \\
D_{1}=\left(\alpha_{\mathrm{t}}^{2}+k_{\|}^{2}\right)^{2}-4 k_{\|}^{2} \alpha_{\mathrm{t}} \alpha_{1}+\frac{\rho_{0}}{\rho} \frac{\omega^{4}}{c_{\mathrm{t}}^{4}} \frac{\alpha_{1}}{\alpha_{0}} \operatorname{coth} \alpha_{0} a
\end{gathered}
$$

and

$$
D_{2}=\left(\alpha_{\mathrm{t}}^{2}+k_{\|}^{2}\right)^{2}-4 k_{\|}^{2} \alpha_{\mathrm{t}} \alpha_{1}+\frac{\rho_{0}}{\rho} \frac{\omega^{4}}{c_{\mathrm{t}}^{4}} \frac{\alpha_{1}}{\alpha_{0}} \text { th } \alpha_{0} a
$$


With these notations, the reflected (in the solid at $x_{3}<a$ ) wave has the following components

$$
\begin{aligned}
& U_{\mathrm{R} 1}=\frac{i k_{\|}}{\alpha_{1}} B_{1} \mathrm{e}^{-\alpha_{1}\left(x_{3}-a\right)}+B_{3} \mathrm{e}^{-\alpha_{\mathrm{t}}\left(x_{3}-a\right)}+ \\
& \quad+B\left(\frac{1}{D_{1}}+\frac{1}{D_{2}}\right)\left[\left(\frac{\omega^{2}}{k_{\|}^{2}}-2 c_{\mathrm{t}}^{2}\right) \mathrm{e}^{-\alpha_{1}\left(x_{3}-a\right)}+2 c_{\mathrm{t}}^{2} \varepsilon \mathrm{e}^{-\alpha_{\mathrm{t}}\left(x_{3}-a\right)}\right] ; \\
& U_{\mathrm{R} 3}=-B_{1} \mathrm{e}^{-\alpha_{1}\left(x_{3}-a\right)}+\frac{i k_{\|}}{\alpha_{\mathrm{t}}} B_{3} \mathrm{e}^{-\alpha_{\mathrm{t}}\left(x_{3}-a\right)}+ \\
& +\frac{i \alpha_{1}}{k_{\|}} \hat{B}\left(\frac{1}{D_{1}}+\frac{1}{D_{2}}\right)\left[\left(\frac{\omega^{2}}{k_{\|}^{2}}-2 c_{\mathrm{t}}^{2}\right) \mathrm{e}^{-\alpha_{1}\left(x_{3}-a\right)}+2 c_{\mathrm{t}}^{2} \mathrm{e}^{-\alpha_{\mathrm{t}}\left(x_{3}-a\right)}\right] .
\end{aligned}
$$

The wave transmitted in the fluid slab $\left(-a<x_{3}<a\right)$ is

$$
\begin{aligned}
& U_{\mathrm{F} 1}=-\frac{\alpha_{1}}{\alpha_{0}} \frac{\omega^{2}}{k_{\|}^{2}} \hat{B}\left[\frac{1}{D_{1}} \frac{\operatorname{ch} \alpha_{0} x_{3}}{\operatorname{sh} \alpha_{0} a}+\frac{1}{D_{2}} \frac{\operatorname{sh} \alpha_{0} x_{3}}{\operatorname{ch} \alpha_{0} a}\right], \\
& U_{\mathrm{F} 3}=\frac{i \alpha_{1} \omega^{2}}{k_{\|}^{3}} \hat{B}\left[\frac{1}{D_{1}} \frac{\operatorname{sh} \alpha_{0} x_{3}}{\operatorname{sh} \alpha_{0} a}+\frac{1}{D_{2}} \frac{\operatorname{ch} \alpha_{0} x_{3}}{\operatorname{ch} \alpha_{0} a}\right] .
\end{aligned}
$$

The wave transmitted in the solid at $x_{3}<-a$ was found to be

$$
\begin{aligned}
& U_{\mathrm{T} 1}=\hat{B}\left(\frac{1}{D_{1}}-\frac{1}{D_{2}}\right)\left[\left(\frac{\omega^{2}}{k_{\|}^{2}}-2 c_{\mathrm{t}}^{2}\right) \mathrm{e}^{\alpha_{1}\left(x_{3}+a\right)}+2 c_{\mathrm{t}}^{2} \mathrm{e}^{\alpha_{\mathrm{t}}\left(x_{3}+a\right)}\right], \\
& U_{\mathrm{T} 3}=-\frac{i \alpha_{1}}{k_{\|}} \hat{B}\left(\frac{1}{D_{1}}-\frac{1}{D_{2}}\right)\left[\left(\frac{\omega^{2}}{k_{\mathrm{f}}^{2}}-2 c_{\mathrm{t}}^{2}\right) \mathrm{e}^{\alpha_{1}\left(x_{3}+a\right)}+2 c_{\mathrm{t}}^{2} \mathrm{e}^{\alpha_{\mathrm{t}}\left(x_{3}+a\right)}\right] .
\end{aligned}
$$

The above results (A.7)-(A.9) also contain the waves corresponding to the localized symmetrical and antisymmetrical modes whose dispersion relation is given by $D_{1}=0$ (Eq. (4.1)) and $D_{2}=0$ (Eq. (4.2)) respectively. The unnormalized wave corresponding to the symmetrical modes $\left(D_{1}=0\right)$ and the antisymmetrical ones $\left(\mathrm{D}_{2}=0\right)$ is obtained by multiplication of the expressions (A.7)-(A.9) by $D_{1}$ and $D_{2}$, respectively, and taking respectively the limit $D_{1} \rightarrow 0$. Note that $\hat{B}$ is in this case a common normalization factor and that for these modes localized within the fluid slab, $\alpha_{1}=\left(k_{\pi}^{2}-\frac{\omega^{2}}{c_{1}^{2}}\right)^{1 / 2}, \alpha_{\mathrm{t}}=\left(k_{\pi}^{2}-\frac{\omega^{2}}{c_{\mathrm{t}}^{2}}\right)^{1 / 2}$ and $\alpha_{0}=-i\left(\frac{\omega^{2}}{c_{0}^{2}}-k^{2}\right)^{1 / 2}$.

\section{Remerciements.}

L'un d'entre nous (L. D.) voudrait exprimer ici sa profonde reconnaissance au Professeur Jacques Friedel pour l'inspiration de départ et son intérêt constant et souriant. En effet le présent travail et plus particulièrement la théorie générale de réponse des interfaces peut être considéré comme une généralisation logique des travaux initiaux du Professeur Jacques Friedel sur les défauts ponctuels.

Trois d'entre nous (L. D., J. M. et A. R.) remercient également le Centre National de la Recherche Scientifique et le Consejo National de Investigationes Cientificas pour leur programme d'échanges. 


\section{References}

[1] LifShitz I. M., J. Phys. U.S.S.R. 7 (1943) 215 and 249 and 8 (1944) 89 ; Usp. Mat. Nauk 7 (1952) 170 ;

Friedel J., Philos. Mag. 43 (1952) 153 ; Ann. Phys. 9 (1954) 158.

[2] Lifshitz I. M. and Rosenzweig L. N., Zh. Eksper i Teor. Fiz. 18 (1948) 1012 ;

Rosenzweig L. N., Tr. Fiz. Otdel. Fiz. Mat. Fakul'teta Khark. Gos. Univ. 2 (1950) 19 ;

See also for example : Dobrzynski L. and Leman G., J. Phys. France 30 (1969) 116.

DobrZynSKi L. and Friedel J., Surf. Sci. 12 (1968) 469.

[3] MASRi P. and Dobrzynski L., Surf. Sci. 34 (1973) 119.

[4] Dobrzynski L., Djafari-Rouhani B. and Hardouin-Duparc O., Phys. Rev. B 29 (1984), 3138, J. Elec. Spec. Rel. Phen. 30 (1983) 119.

[5] Dobrzynski L., Surf. Sci. Rep. 6 (1986) 119 ; Surf. Sci. 175 (1986) 1 ; Surf. Sci. 180 (1987) 489.

[6] Akjouj A., Sylla B., Zielinski P. and Dobrzynski L., J. Phys. C 20 (1987) 6137 ; Phys. Rev. B 37 (1988) 5670 ;

Akjouj A., Zielinski P. and Dobrzynski L., J. Phys. C 20 (1987) 6201 ;

MASRI P. and DOBRZYNSKI L., Surf. Sci. 198 (1988) 285;

Rahmani M. D., Masri P. and Dobrzynski L., J. Phys. C 21 (1988) 4 ;

Szwacka T., Noguera A., Rodriguez A., Mendialdua J. and Dobrzynski L., Phys. Rev. B 37 (1988) 8451.

[7] Dobrzynski L., Surf. Sci 180 (1987) 505 ; Phys. Rev. B 37 (1988) 8027 ; Surf. Sci. 200 (1988) 435.

[8] Garcia-Moliner F. and Rubio J., J. Phys. C 2 (1969) 1789 ;

Garcia-Moliner F. and Flores F., Introduction to the Theory of Solid Surfaces (Cambridge University Press) 1979 ;

Garcia-Moliner F. and Velasco V., Prog. Surf. Sci. 21 (1986) 93 ;

Garcia-Moliner F., Ann. Phys. Fr. 2 (1977) 179.

[9] Dobrzynski L. and Puszkarski H., J. Phys. Condens. Matter 1 (1989) 1239.

[10] Maradudin A. A. and Mills D. L., Ann. Phys. 100 (1976) 262.

[11] Djafari-Rouhani B. and Dobrzynski L., J. Phys. France 43 (1982) 523.

[12] See for example :

Maradudin A. A., Non equilibrium Phonon Dynamics, Ed. W. E. Bron, Nato ASI Ser. B Phys. 124 (1986) 395.

[13] DAMIEN J. C., private communication.

[14] Velasco V. R. and Djafari-Rouhani B., Phys. Rev. B 26 (1982) 1929 ;

Velasco V. R., Djafari-Rouhani B., Dobrzynski L. and Garcia-Moliner F., Le Vide les Couches Minces Suppl. Vol. II 201 (1980) 774.

[15] Wendler L. and Grigoryan V. G., Surf. Sci. 206 (1988) 203.

[16] Djafari-Rouhani B. and Dobrzynski L., Solid State Commun. 62 (1987) 609 ;

Mendialdua J., Szwacka T., Rodriguez A. and Dobrzynski L., Phys. Rev. B (1989) in press.

[17] Deymier P., Janot L., Li J. and Dobrzynski L., Phys. Rev. B 39 (1989) 1512.

[18] See for example :

EWING W. M.., JARDETZKY W. S. and Press F., Elastic Waves in Layered Media (Mc Graw-Hill Pub., New York) 1957 ;

MÜller G., J. Geophys. 58 (1985) 153.

[19] See for a review : CotTAm M. G. and MARAdudin A. A., Surface Excitations, Modern Problems in Condensed Matter Sciences (North-Holland, Amsterdam) Vol. 9 (1986).

[20] Deymier P. et al., to be published. 